Horizons philosophiques

\title{
Habiter en kosmopolite : enquête sur les modes de comportement
}

\section{Angela Cozea}

Volume 17, numéro 2, printemps 2007

La pensée de Peter Sloterdijk

URI : https://id.erudit.org/iderudit/802638ar

DOI : https://doi.org/10.7202/802638ar

Aller au sommaire du numéro

Éditeur(s)

Collège Édouard-Montpetit

ISSN

1181-9227 (imprimé)

1920-2954 (numérique)

Découvrir la revue

\section{Citer cet article}

Cozea, A. (2007). Habiter en kosmopolite : enquête sur les modes de comportement. Horizons philosophiques, 17(2), 81-107.

https://doi.org/10.7202/802638ar d'utilisation que vous pouvez consulter en ligne.

https://apropos.erudit.org/fr/usagers/politique-dutilisation/ 


\section{Habiter en kosmopolite: enquête sur les modes de comportement}

Le 8 juin 1931, Walter Benjamin raconte dans son journal «un après-midi vraiment mémorable avec Brecht". II s'agit d'une "enquête» engagée, à la suggestion de Benjamin, sur les modes de comportement, sur la question de l'habitation, qui est son sujet de prédilection, et surtout sur le mode qui donne à l'habitant le minimum d'habitudes, "l'installation de fortune". II s'agit d'un mode d'habitation destructeur, "qui ne laisse se former aucune habitude parce qu'il se débarrasse progressivement des choses qui en sont les points d'appui». Ce qui tourmente Benjamin, de son propre aveu, la raison pour laquelle il veut en parler sans la présenter comme «la sienne propre», est que "ce sont précisément les caractères permanents de la situation des écrivains qui sont désolants, mais, pour des raisons touchant à l'honneur de la profession, ils ne sont presque jamais exposés entièrement au grand jour».1

Je propose une lecture de certains textes de Peter Sloterdijk à la lumière de ces difficultés à caractère permanent qui marquent la condition de l'écrivain, telle qu'exposée par Benjamin dans son journal. Il s'agira, bien sûr, de réfléchir à ce qui, au sein de cette condition, est révélateur du monde où nous vivons en tant que kosmopolites aujourd'hui, en tant que géniteurs et enfants de l'individualisme post-politique : "la signification de ce terme est largement illustrée par notre époque où les villes sont remplies d'individus isolés qui, bon gré mal gré, n'appartiennent à personne ermites bourgeois, originaux sans attaches, orphelins de la psyché, fous de travail enfermés dans leur bureau, célibataires cosmiques".?

Mon commentaire tourne essentiellement autour de trois aspects qui marquent la condition de l'écrivain du début du $X X X^{e}$ siècle d'un coté, de l'individu entraîné par les médias égotechniques de la fin du $X X$ de l'autre : habiter - ou non - le territoire du langage, penser l'habitation et communiquer - ou non - avec les morts.

\section{L'installation de fortune}

Le journal ${ }^{3}$ de 1931 de Walter Benjamin dont il sera question ici commence par un commentaire sur la situation de l'écrivain de son 
temps. Les scènes auxquelles je vais m'arrêter portent sur l'accomplissement des vœux et sur le don de fleurs - sur l'extase du solitaire qui ramène de sa promenade des fleurs en guise de remerciement même si Benjamin est le premier à se demander si cela peut encore signifier quelque chose : l'extase de cet ordre est-il encore - tout comme "la fleur bleue» - de saison?

Le théâtre - soit l'état d'âme - où les dites scènes se déroulent est la disposition grandissante de l'écrivain à se suicider, ou plutôt : "ma promptitude grandissante à m'enlever la vie» : "Et pour ne parcourir entièrement que le cercle des pensées et des forces sous la commande desquelles ce journal est venu à être entamé, il ne me reste plus qu'à évoquer ma disposition (Bereitschaft) grandissante à m'enlever la vie.» (ÉA 175; traduction légèrement remaniée)

À Juan-les-Pins, le 4 mai 1931, Benjamin se trouve encore une fois éprouver un sentiment de répulsion croissant à l'égard "des chemins" où il voit des hommes, dans la même position et "de la même espèce" que lui, "s'engager en Allemagne pour maîtriser la désolante situation politico-intellectuelle». II est tourmenté par «les caractères permanents de la situation des écrivains", qui sont désolants, mais qui, "pour des raisons touchant à l'honneur de la profession, ne sont presque jamais exposés entièrement au grand jour». Est-ce que cette disposition pacifique particulière ne se rattache-t-elle pas, se demande l'auteur, "à cet esprit de contemplation auquel vous fait accéder l'usage des drogues"? (ÉA 174)

L'écrivain qui observe les choses telles qu'elles se présentent à ce moment-ci en Europe se retrouve dans la même situation, amère, que le drogué vis-à-vis ses congénères. Il est pacifique, il est contemplatif, mais cette attitude pacifique et contemplative est l'attitude de celui qui sent qu'il ne peut plus pénétrer l'ordre qu'il observe.

Rêver de la fleur bleue, ce n'est plus de saison. Pour se réveiller aujourd'hui dans la peau d'Heinrich von Ofterdingen, il faut avoir oublié l'heure. L'histoire du rêve reste encore à écrire, et l'étude historique, en mettant ce domaine en lumière, ouvrirait une brèche décisive dans la superstition d'une détermination naturelle des phénomènes humains. Le rêve participe à l'histoire. [...] Le rêve n'ouvre plus sur les lointains d'azur. II est devenu gris. Les rêves sont à présent des chemins de traverse menant au banal. La technique confisque définitivement l'image extérieure des choses, comme des 
billets de banque qui vont être retirés de la circulation. Dans le rêve, la main s'en saisit une dernière fois, elle prend congé des objets en suivant leurs contours familiers. Elle les saisit par l'endroit le plus usé. 4

II existe un savoir qui nous est caché dans la vie ainsi dite réveillée; le savoir essentiel, celui qui nous conduit et qui détermine notre destinée, est un savoir auquel nous n'avons accès qu'à travers un lent processus de rêve et d'écriture. L'acte de copier - que Benjamin a toujours pratiqué, mais qui s'avouera essentiel lorsqu'il entame la rédaction du Livre des passages, dont il sera question plus loin - en fait partie intégrante, car copier signifie "recopier» pour nous-même quelque chose qui gît dans ces chambres intérieures de notre être, arrangées, organisées conformément à l'expression : la disposition de notre paysage imaginaire. tre tourné vers le passé ouvre la scène de notre sensibilité devant le paysage, de nos expériences précoces de l'espace. ${ }^{5}$ Benjamin définit cette ouverture en tant que mouvement de submersion : vertiefen, approfondir. Paysage imaginaire parce que désiré, et dont il dira, quelques jours plus tard:

Voici ce que veut le rêveur abîmé dans le paysage : [que] la mer [fasse] gonfler et retomber à chaque instant des milliards et des milliards de vagues, [que] les forêts frémissent de nouveau à chaque instant des racines jusqu'à la plus haute feuille, [qu']en un mouvement ininterrompu les pierres du château s'effritent et tombent, [...]. (AS 427-8)

La science ne veut voir dans les atomes que des tempêtes d'électrons, alors que le rêveur abîmé dans le paysage doit oublier tout cela «pour s'abandonner aux images auprès desquelles <il> veut trouver la paix, l'éternité, le calme, la durée." (ÉA 182) Même si un moustique qui bourdonne à ses oreilles, un coup de vent qui le fait frissonner, toute proximité qui l'atteint donne le mensonge à cette illusion de paix, d'éternité, de calme, de durée...

En traversant ce paysage imaginaire, je voudrais approcher la deuxième scène, de la pivoine, afin de concevoir le passage de l'objet offert à sa signification qu'on appelle "conceptuelle». Mais, pour ne pas succomber à l'emprise d'un processus de conceptualisation où les pensées se retrouveraient coupées et ainsi "délivrées" de leur incarnation dans le monde, la lecture nous sera utile, d'une lettre de Wiesengrund-Adorno à Benjamin, (Berlin, 6.9.1936) par l'entremise 
de laquelle, tout en lui adressant "quelques mots rapides sur le travail consacré au Narrateur", Adorno reproche à son aîné de faire du corps humain la mesure de la concrétude :

Je commencerais par vous assurer de mon accord plein et entier avec l'intention historico-philosophique : à savoir, la narration n'est plus possible. [...] En revanche, je ne m'inscris pas en conformité avec la tendance à réduire le geste de l'immédiateté [...] non pas tant à l'immédiateté au sens hégélien, au sens historico-philosophique, qu'au geste au sens somatique. Et cette divergence me conduit au centre de notre discussion comme rarement. Car, en dépit de l'accord le plus fondamental et le plus concret par ailleurs, tous. les points sur. lesquels je diffère de vous pourraient se résumer sous le titre d'un matérialisme anthropologique, que je ne peux suivre. C'est comme si pour vous le corps humain était la mesure de la concrétude. Or il n'est qu'un «invariant», de telle nature, je crois, qu'il barre la route au concret décisif (''image dialectique précisément, et non l'image archaïque). C'est pourquoi je me sens toujours mal-à-l'aise en vous voyant utiliser des termes comme geste et autres choses du même genre [...].6

Le sens de la correction qu'Adorno apporte à l'essai de Benjamin est de faire accepter à son ami que la dimension corporelle de la connaissance - comme de la représentation d'ailleurs - ne devrait être qu'un «invariant», et non pas l'élément essentiel de sa pensée; un invariant parmi d'autres, insiste Adorno, qui, plutôt que de nous renseigner sur le sens de l'histoire, nous empêche, justement, d'en saisir le sens. Ce qui, dans le rapport à l'Histoire, est de l'ordre de (l')immédiateté ne peut être conçu comme tel que si le mot est pris dans son sens hégélien, "au sens historico-philosophique», et non point en tant que "geste au sens somatique». Lorsqu'il fait cette lecture et cette correction, Adorno dévoile non pas seulement l'entrave que sa jeunesse pose à sa pensée, mais la manière dont la pensée elle-même peut, si elle ne prend garde, prendre une certaine forme de jeunesse de la pensée - qu'elle imagine, parfois, impérissable - pour de la rigueur conceptuelle. ${ }^{7}$

Ainsi, pour revenir à la question du passage du concret à l'abstrait chez Benjamin, il nous faudra retrouver plutôt notre rêveur abîmé dans le paysage : «Et il semble parfois connaître l'accomplissement 
quand il parvient à désamorcer le mouvement même, à métamorphoser le tremblement des feuilles au-dessus de lui en cime, le passage rapide des oiseaux autour de sa tête en migration.»8 (ÉA 183)

Est-ce que le rêveur recherche l'immobilité du concept? Plutôt celle de l'éternité, il me semble. La traduction nomme cette immobilité, cristallisation : "Circonscrire ainsi la nature au nom d'images pâlies c'est la magie noire de la sentimentalité. Mais la faire cristalliser par une nouvelle invocation, c'est le don du poète." (ÉA 183) II se peut que le mot "cristallisation" ait été employé pour donner à l'expression une tournure poétique; il n'en reste pas moins que le verbe que Benjamin choisit ici est erstarren : devenir raide, se raidir, comme dans l'expression im Tode erstarren die Glieder : la mort raidit les membres.

Dans son essai sur le Narrateur, achevé, comme la lettre d'Adorno l'atteste, en 1936, Benjamin conçoit la destinée d'un homme mort jeune comme suit : «Un homme qui est mort à trente-cinq ans [...] apparaît dans la remémoration (Eingedenken), à chaque point de sa vie, comme un homme qui devait mourir à trente-cinq ans." ${ }^{9}$ Dans son journal de 1931, il décrivait déjà son sentiment d'avoir vécu une vie dont les vœux les plus chers avaient été exaucés. Le même 4 mai 1931, il écrit :

Les gens mettraient moins en doute la proposition selon laquelle chacun voit ses vœux les plus profonds exaucés s'ils se disaient que ces vœux sont presque toujours inconscients (unbewußt), en d'autres termes différents de ceux qu'ils connaissent (denen sie wissen) et dont ils peuvent à bon droit se plaindre que l'exaucement leur ait été refusé. [...] Mais le véritable signe distinctif de ces vœux était qu'aucun prix n'était trop élevé pour ce que l'on désirait : peut-être d'ailleurs reconnaît-on le mieux ses vœux les plus chers, rétrospectivement, en voyant quel bon usage ils ont fait de cette disposition illimité à payer et quel prix il a fallu payer pour les exaucer. (ÉA 175-6; AS 423)

Le 3 juin, à la Potinière, dans le Lavandou, Benjamin passe quelques heures avec Brecht et d'autres amis; «il y aurait toutes sortes de notes à prendre sur les conversations avec Brecht", confesse-t-il : 
Nous avons abordé les sujets les plus divers : la société internationale des amis matérialistes de la dialectique hégélienne; l'idée d'un drame policier; le procès de Friedrich Schiller; et même, pour finir - c'était hier - une conversation d'une heure, $[. .$.$] sur Proust. Mais je préfère raconter une autre$ scène parce que mon comportement, tel qu'il s'est révélé à cette occasion, est vraiment impénétrable. (ÉA 187-189)

Cet aspect impénétrable enveloppe ce que j'appelle la scène de la pivoine, scène ou histoire dont la narration pourrait aussi bien commencer par la fille en veste rouge et pantalon bleu que Benjamin avait poursuivi ce jour-là - car il dit : «J'avais fait une promenade solitaire vers Saint-Clair. C'était la première fois depuis longtemps, la première promenade en solitaire, depuis longtemps." (ÉA 187-189) Depuis longtemps il n'avait fait une promenade solitaire, depuis longtemps peut-être n'avait-il poursuivi une jeune fille en veste de plage rouge et pantalon bleu, mais auxquelles promenade et poursuite une fin abrupte fut mise par la rencontre, au carrefour du chemin menant à la maison de Brecht, d'un homme que la jeune fille connaissait :

Chemin faisant, comme je passai devant la villa Mar-belo où Brecht et les autres vivaient, j'ai eu envie de monter les voir. Et bien que je me sois dit qu'ils devaient être encore à table, c'est ce que j'ai fait; et dans l'état un peu flageolant où m'avait mis cette promenade solitaire, la première depuis si longtemps, la moindre des raisons de ma décision n'était sans doute pas que j'étais fátigué de suivre une jolie jeune fille, vêtue d'une veste de plage rouge et d'un pantalon bleu, qui marchait devant moi sur la grand-route dans le crépuscule. Le pire, c'est qu'elle rencontra tout à coup un homme et s'arrêta auprès de lui et qu'il m'aurait fallu passer à côté d'elle. Je pris alors sur le côté le chemin qui menait à la villa et j'entrai dans le vestibule. (ÉA 188)

Pendant sa promenade, il avait cueilli des fleurs : une églantine et une pivoine. Heckenrose et Pfingstrose. Cette pivoine, ou rose de la Pentecôte, comme son nom signifie en allemand, lui rappelle le visage d'un amour lointain déjà : "Sur le chemin de retour, je passai devant un buisson de pivoines. Elles me rappelèrent avec force le bouquet que Jula $<$ Cohn $>$ m'avait offert il y a de nombreuses années 
pour mon anniversaire et qui n'était composé que de pivoines; j'en ai cueilli avec peine un petit rameau que je plaçai avec l'églantine entre les pages du Journal du coiffeur de Jouhandeau que j'avais avec moi.» (ÉA 188)

Avec son livre et les fleurs, il passera donc visiter Brecht. Ils restèrent, soit seuls, soit en compagnie des autres, deux heures environ, à converser, jusqu'à ce que le moment lui sembla venu de partir. Alors qu'il reprenait son livre,

les fleurs en dépassaient et lorsqu'on les montra en faisant des plaisanteries, mon embarras a été d'autant plus grand qu'avant d'entrer dans la maison je m'étais demandé à quoi rimait d'entrer avec des fleurs (was das Erscheinen mit Blumen denn solle) et si je ne ferais pas mieux de les jeter. Mais je ne l'avais pas fait, Dieu sait pourquoi; certainement il y avait eu en ce moment-là un sentiment de défi (Trotz). [...] Je fis ironiquement cadeau de la pivoine à Brecht, en subissant ses plaisanteries ironiques, conservant encore l'églantine; mais naturellement il ne l'accepta pas. (AS 432)

Que viennent donc chercher ces roses, et puis des fleurs bleues encore, là-dedans? II fit disparaître ses roses en fin de compte dans un grand pot plein de fleurs bleues qui se trouvait à côté de lui, il fit "tomber l'églantine parmi les autres fleurs du pot où elle sembla, curiosité botanique, partir de la tige d'une des fleurs bleues". Curiosité botanique et couronnement, sinon conclusion d'une bien grave conversation intellectuelle concernant des sujets parmi les plus sérieux, comme la société internationale des amis matérialistes de la dialectique hégélienne, l'idée d'un drame policier, le procès de Friedrich Schiller, bref, conversation ramassant une foule d'éléments, apparemment disparates, mais que l'on sait parfaitement en mesure de participer de ce schéma qui ne cesse d'être formulé entre les amis matérialistes, et qui vise la mise en place d'une réflexion sur l'histoire à laquelle tout penseur sérieux devrait, nécessairement, contribuer. D'un autre côté, que deviennent la pivoine, l'églantine, lorsqu'on les regarde dans l'esprit de la contemplation du rêveur abîmé dans le paysage, contemplation pour laquelle les feuilles tremblantes se métamorphosaient en cime, le passage des oiseaux devenait migration?

Les conversations que Benjamin a avec Brecht tournent souvent autour des conditions de vie de Benjamin lui-même, mais toujours à 
l'intérieur de contraintes imposées par ce que ce dernier appelle «'honneur de la profession». Des sujets comme l'enquête sur les modes de comportement, où la proposition de Benjamin «rejoignait [s]on sujet de prédilection, la question de l'habitation", sujets instructifs s'il y en a, eu égard aux difficultés financières ou plutôt alimentaires que Benjamin traverse concrètement, ne sont pas traités de manière littérale, mais par des figures qui leur servent d'intercesseurs. S'agirait-il ici, encore une fois, de ce que l'on appelle des «figures de la pensée» tout simplement? Benjamin au sujet de I'habitation, Benjamin assumant son rôle de visiteur qui tend la rose de la Pentecôte à Brecht - est-ce que ces figures sont des figures de la pensée uniquement?

Le 8 juin, pendant un après-midi mémorable avec Brecht, Benjamin, en refusant de poursuivre avec son ami une autre "recherche" encore sur les "conceptions", réclame, à la place, une "enquête sur les modes de comportement".

Brecht [...] nous fit une extraordinaire présentation de sa manière d'habiter que je mis ensuite en parallèle avec une autre - que je n'ai pas présentée comme la mienne propre. [...] Brecht est parti de son mode d'habitation "réciproque» $<$ mitahmends. C'est un mode d'habitation qui façonne l'environnement et l'aménage à sa mesure, de façon souple et adaptée; un mode où celui qui habite est à sa manière chez lui. II mit ce mode en parallèle avec son autre mode d'habitation, une attitude qui consiste à ne se sentir partout que comme un invité; puis il a décliné toute responsabilité vis-à-vis ce qui lui est utile; il se sent comme invité par le fauteuil où il prend place et, à un moment donné, il se sent congédié. (ÉA 193-194)

Tout en distinguant le mode d'habitation qui donne à l'habitant le maximum d'habitudes et celui qui lui en donne le minimum, Benjamin insiste sur le fait que les deux extrêmes sont pathologiques. "Le mode qui donne à l'habitant le maximum d'habitudes est celui que se représente la logeuse. L'homme devient fonction des opérations que les accessoires exigent de lui. Ici règne une relation de l'habitant avec le monde des choses <Dingwelt> tout autre que dans le mode "réciproque" d'habiter." Alors que dans ce dernier mode, défendu par Brecht, Benjamin voit les choses rendre «à peu près les services d'un dispositif scénique", et celui qui y loge, habiter un dispositif, dans le 
mode permettant le maximum d'habitudes, les choses sont prises au sérieux, et celui qui y loge habite un intérieur. Quant à déterminer le facteur de l'habitude dans le mode d'habitation "réciproque", la chose est moins aisée; par contre, pour celui qui habite comme un invité, «le facteur est parfaitement défini par Nietzsche : "J'aime les courtes habitudes."»

Le quatrième mode d'habitation enfin, celui qui donne à l'habitant le minimum d'habitudes, est l'installation de fortune. Cette conception elle aussi trouve son expression la plus élaborée dans la générosité de la logeuse. En son centre il y a le mauvais locataire et les dégradations. Car l'installation de fortune est le mode d'habitation destructeur, le mode d'habitation qui ne laisse se former aucune habitude parce qu'il se débarrasse progressivement des choses qui en sont les points d'appui. (ÉA 194-195)

\section{Voyageurs sans correspondance immédiate}

Pour comprendre les sources du malaise profond que Benjamin ressent, par rapport à la condition de l'écrivain de son temps, il nous faudra accepter, avec Sloterdijk, que le principal événement logique de notre époque est «l'expulsion hors des habitudes de l'apparence humaniste". Le domaine de l'histoire de l'esprit et celui de la technique partagent une caractéristique commune : le fait que la culture technologique produit un nouvel état d'agrégat du langage et du texte qui n'a plus rien de commun avec ses interprétations traditionnelles par la religion, la métaphysique et l'humanisme. "La "maison de l'Être" se révèle comme une chose dans laquelle un séjour, au sens de l'habitat et du rapprochement du lointain, n'est pratiquement plus possible." La parole et l'écriture n'ont plus de sens qui soit domestique d'une manière ou d'une autre. Les compositions de la technique, plutôt que de susciter l'apprivoisement de l'extériorité, «augmentent au contraire le volume de l'extérieur et du jamais assimilable. La province du langage se réduit [...].»10

Le rapport est, indéniablement, serré et substantiel, entre ces deux "modes de comportement»: habiter la province du langage, penser l'habitation. Dans Écumes, Sloterdijk affirme que «tout le savoir que nous portons en nous sur les logements et les habitudes de l'ancien fonds reflète un habitus, celui du statut d'habitant sur des terres natales, dans des patries et dans des régions." La vie dans 
cette sédentarité "triomphale» était «trop lente, trop repliée sur elle-même et trop inspirée par le modèle végétal» (É 447) pour pouvoir réfléchir de manière appropriée à sa propre constitution. $\mathrm{Ce}$ qui caractérise la connaissance théorique - la déterritorialisation instaurée avec les nouvelles conditions de transport modernes, nous permet de repenser l'habitation contemporaine comme un lieu "de halte protégée et de salle d'attente climatisée», voué à des «voyageurs sans correspondance immédiate» (É 448).

La recherche des origines de tels voyageurs nous ferait descendre loin dans l'histoire, nous ferait aller chercher, dans la figure des sages et des saints, la disposition à être "seul avec l'absolu, représenté comme la totalité et l'universel. Ces individus s'entraînent à l'exil pour tester leur capacité à vivre pleinement dans n'importe quelle communauté, [...] en évitant pourtant tout véritable échange, toute relation affective." Si dans cette robustesse il y a quelque chose d'inhumain, on y reconnaît aussi, en creux, la capacité à s'investir, la solitude mobilisée par "une vie passée dans l'excellence». (MB 52)

Qui se qualifie de kosmopolites se situe déjà au-delà de la ville et table sur un monde où l'appartenance à des communautés substantielles pourrait un jour prendre fin. C'est un vagabond spirituel de l'ancienne Athènes qui inventa le mot servant maintenant à désigner l'individualisme post-politique en Europe; la signification de ce terme est largement illustrée par notre époque où les villes sont remplies d'individus isolés qui, bon gré mal gré, n'appartiennent à personne - ermites bourgeois, originaux sans attaches, orphelins de la psyché, fous de travail enfermés dans leur bureau, célibataires cosmiques. (MB 53)

Seul celui qui s'est exercé à se séparer de ce qui lui est proche peut représenter l'abstrait, c'est-à-dire, figurer et représenter le pouvoir, communiquer et faire apprendre aux marges la «langue mondiale» qui est la langue du centre. (MB 51) D'un côté, il faut compter avec la violence de l'inéluctable qui bouscule les individus prêts dorénavant à passer leur vie à exécuter les tâches qu'il leur incombe d'exécuter. De l'autre, il faut oublier «les laborieuses synchronisations héritées du passé et qui, dans la politique classique, reliaient les formes de l'âme et celles du monde," car elles ne suffisent plus, quand l'enjeu est celui de l'existence dans un monde global. "Le bon vieux cosmopolitisme se transforme en un nomadisme cosmopathique [...].» (MB 57-58) 
Le fait que l'homo sapiens, animal fait pour vivre en petits groupes, est dépassé par les exigences de la grande civilisation, se manifeste dans une forme particulière d'impuissance : il ne réussit plus à créer des prothèses symboliques et émotionnelles lui permettant d'évoluer dans le Grand. (MB 66) Dans cet univers, devant I'horizon unitaire des temps modernes, l'espèce orpheline a néanmoins essayé de formuler un autre principe d'appartenance : "quelque chose est mort ou est voué plus ou moins à la dégénérescence, mais la vie et la civilisation n'en continuent pas moins d'avancer, d'une façon ou d'une autre». Réfléchir sur «la troisième période" 11 signifie comprendre et accepter la perte de l'esprit de l'ère agraire et ses concepts spatiaux devenus caduques face à un univers synchrone : "[l]es acteurs du nouveau jeu mondial de l'ère industrielle ne se définissent plus par rapport au sol et à la "patrie" mais par des accès aux gares [...] et à toutes sortes de possibilités de raccordement". (MB 56-57) On peut également présenter cette opposition entre le sol de la patrie et les gares qui nous permettent de décoller vers le partout et le nulle part en termes du conflit gigantesque qui court à travers le monde moderne : le conflit entre les protecteurs de la vie et les consommateurs de la vie. En termes typologiques, il s'agit du conflit entre l'habitus paysan et l'habitus nomade :

Le dilemme éthique des modernes tient au fait qu'ils pensent comme des végétariens et vivent comme des carnivores. C'est la raison pour laquelle l'éthique et la technique, chez nous, ne peuvent jamais courir en parallèle. Nous voulons être aussi bons que les bons pâtres, mais vivre aussi bien que les mauvais pâtres qui sont tristement fameux pour leurs fêtes d'abattage et les orgies au cours desquelles ils gaspillent la vie. Ce dualisme fait que tous les débats actuets sur l'éthique ont un ton déplacé. L'homme moderne parle un double langage, c'est un nomade portant la peau d'un mouton, ou le mauvais berger dans l'habit du bon : un consommateur final. Quand on rencontre aujourd'hui cet éloge du nomade qui est devenu intellectuellement populaire, il faudrait se rappeler qu'avec lui, c'est [...] le mode d'existence des nomades pastoraux qu'on réhabilite - que personne n'aille croire qu'il s'agit d'un discours innocent. ${ }^{12}$ 
Le dilemme éthique reste un dilemme avec lequel il faut vivre, qu'il faut accepter et, d'une façon ou d'une autre, perpétuer. La tâche de cette lucidité désenchanteresse, au caractère viril et tonifiant, est d'engendrer une parole qui dévoile le secret asséché de l'île humaine. Ce qui n'empêche que, au cœur de ce dégrisement, la confession d'une certaine limite se fasse simultanément entendre, selon laquelle notre île reste un lieu hanté par la vie révolue. (É 389) Deux sources abreuvent de leur eau-de-vie le souci des mortels pour l'absent ou le transcendant : l'ordre ou la loi ontologique, qui nous fait constater que notre pensée et notre savoir actuels "constituent une île dans la mer d'une pensée et d'un savoir plus grands"; qui nous fait accepter que l'intelligence n'existe que dans la dénivellation, et qui «s'exprime avant tout à travers le fait qu'elle prend pour point de repère ce par quoi elle se voit surmontée». Et l'ordre ou la loi du langage qui nous vient des premiers Grecs, et nous donne le nom de "mortels". Non pas seulement dans le sens où la mort nous attend, mais, plus encore, dans le sens que nous avons nos morts derrière nous. Le culte - qui fait venir les morts de manière ordonnée - et l'apparition du spectre - qui permet aux morts de réapparaître de manière dérégulée - ont en commun le fait de mettre en scène la relation entre transcendance et lieu. Un des aspects de cette théorie qui cherche à démontrer en quoi les concepts mûris au fur de l'ère agraire sont devenus caduques est l'évidence, logique apparemment, selon laquelle on ne peut pas pratiquer le culte des ancêtres ailleurs qu'à proximité des lieux qu'on avait en commun avec eux. Les voyageurs sans correspondance immédiate, les kosmopolites, ne se sont pas seulement coupés affectivement de leurs proches; ils s'en sont avant tout éloignés dans l'espace, ils ont quitté la patrie.

Le journal de Benjamin, sa correspondance avec Adorno, ainsi que sa Chronique berlinoise, montrent comment ce processus d'éloignement se déroulait à l'un de ses tournants les plus dangereux. Dans son journal par exemple, en relatant un dialogue entre Brecht et Brentano sur la question du «ralliement des travailleurs intellectuels à la révolution", Benjamin note la virulence avec laquelle Brecht pose la question : est-ce que la situation de l'intelligentsia est-elle à proprement parler mauvaise?

L'intelligentsia, dit-il, ne se surmène en aucun cas. S'il y a quelques médecins, ou quelques avocats qui bossent, et alors? C'est un travail qui leur convient, on ne peut sous aucun prétexte le comparer à celui du prolétaire. Et pour finir les gens 
se demandent ce qu'ils vont faire à soixante ans, s'ils n'ont rien mis de côté - ah oui, cria-t-il, tout à fait exaspéré, c'est vraiment trop demander. Et alors, nom de Dieu, ils crèvent. Beaucoup trop tard, ils feraient mieux le faire dès maintenant. (ÉA 193)

Benjamin, Adorno, Brecht vivaient ces années ' 30 - ces années de la défaite d'un monde - chacun à sa manière, et pourtant, leur rapport à leur travail, à leur écriture et à leur pensée, restait d'autant plus âpre. Si dans le travail de l'intellectuel d'aujourd'hui détermination il y a, et peut-être y reste-t-il un semblant de détermination, on peut se demander jusqu'à quel point l'intellectuel cosmopolite est encore capable de se plier à cette discipline qui les assujettissait, eux, sans répit. Il est certain que le renversement de rôles entre Benjamin et Adorno est difficile à témoigner; les commentaires d'Adorno, insupportables; son attitude devant cet ami terrassé par le sort, hautaine et humiliante. Mais en même temps on n'aurait peut-être pas tort de soupçonner que Benjamin lui-même savait, intimement, inconsciemment ou secrètement, que rien ne lui restait à part cette acharnée dispute au sujet de ce qui, dans leur travail, les séparait, de ce qui les rapprochait.

Ce qui est en jeu pour nous, à l'époque contemporaine, est bien plus de l'ordre de ce que Sloterdijk appelle "le jeu de l'autocomplément des individus devant le miroir (et devant les autres médias de l'ego-technique, notamment le livre, celui à lire et celui à écrire)". II s'agit d'un processus contribuant à la

fiction de l'autonomie - ce rêve de la domination sur soimême qui, depuis les commencements de la philosophie antique, s'introduit dans l'image dominante de la vie sage. Parce qu'il se reconnaît soi-même, le sage qui peut être son propre maître n'est pas forcément traversé par un regard de souverain, il n'a même plus aucune obligation d'être fixé par le regard de l'autre. II posséderait ainsi une qualité à laquelle Hegel, sur un ton triomphal, a donné le nom d'impénétrable.

Le chemin est donc court, du "Connais-toi toi-même" au "Complète-toi toi-mêmẹ". (B 221)

Le cosmopolite d'aujourd'hui a cessé de se faire des amis; pourquoi, on peut se demander, à l'intérieur de l'université, aucune amitié n'est plus possible pour nous? 
Ces intellectuels, ces cosmopolites-là, que nous appelons amis sans trop savoir ce que cela voulait dire pour eux, se soumettaient à des principes dont ils ne partageaient pas toujours le contenu, mais la nécessité. Et puisque Benjamin lui-même appelle cette nécessité, I'honneur de la profession, nous pouvons supposer qu'il s'agissait d'un certain code d'honneur du philosophe, si je peux m'exprimer ainsi. La discussion sur l'habitation de fortune, avec Brecht, où Benjamin ne dévoile pas qu'il s'agit de son cas, même si tous ceux qui écoutent autour de lui le comprennent implicitement; le va-et-vient des échanges épistolaires autour des remarques d'une connaissance commune, qui avait demandé à Adorno de s'occuper de Benjamin car il était démuni (Else H., 2 juin 1936), intervention qui met les deux amis dans une position très pénible et suite à laquelle Benjamin ne cesse de s'excuser pour le malentendu, me font penser à combien nous sommes éloignés de cet «honneur de l'intellectuel».

Un sentiment d'irréversibilité accompagne l'éloignement de la ville natale. Dans Chronique berlinoise, Benjamin évoque la difficulté de savoir jusqu'où on peut remonter dans ses souvenirs :

Que ma sensibilité pour cette tradition de la ville de Berlin ... pour cette tradition topographique qui représente le lien avec les morts ensevelis sous ce sol, ait ses frontières, voilà qui était déjà décidé par le simple fait.que les familles de mes deux parents n'étaient pas autochtones. Cela fixe une frontière aux souvenirs de l'enfant - davantage que le contenu même de ses expériences dont rendent compte les pages qui suivent. (ÉA 282)

Sloterdijk ne manque pas d'observer, lui aussi, certains écarts par rapport à la certitude que dessine sa théorie de l'éloignement. II prend dûment note des exceptions à la règle selon laquelle les individus entraînés à l'exil arrivent à croire à leur capacité à vivre pleinement dans n'importe quelle communauté. II admet que le culte des ancêtres pratiqué par les émigrés témoigne de la possibilité d'une relation déterritorialisée avec eux. (É 390) Mais il finit par faire, de cette déterritorialisation et hantise, un autre trait encore du cosmopolitisme moderne : les morts seraient, eux aussi, au fait de la mondialisation, et ceux d'entre eux qui n'auraient pas trouvé la paix auront appris à penser globalement et à hanter localement. $(E ́ \text { 391 })^{13}$

Si étonnante et peu vraisemblable qu'elle puisse paraître, cette hantise des spectres cosmopolites appartient néanmoins à l'une des formes les plus anciennes de la grammaire de l'appartenance : 
Elle définit des catégories et des attitudes différenciées entre jeunes et vieux, hommes et femmes; elle édicte des règles et des rapports entre "individus" et "étrangers", aussi bien qu'entre vivants et morts, et même entre vivants et non-nés. L'invention universelle du culte des ancêtres ouvre la voie à une pensée protométaphysique - comme si partout les ancêtres morts étaient ceux qui commandaient la pensée, comme l'enseignait Heidegger pour qui penser équivalait à remercier (les morts - bien qu'il ne l'ait pas formulé de façon aussi explicite). (MB 25)

Le fait que, de nos jours, on ne remercie plus les morts, ${ }^{14}$ confirme en premier lieu le fait que "les grandes civilisations détournent leur attention de la répétition de l'homme par l'homme pour s'interroger d'abord sur l'utilisation de l'homme par l'homme". (MB 25)

Le processus de la mise en absence des morts est lié à la terreur qu'inspirait aux humains la méchanceté des dieux, et plus encore peut-être la tendance des ancêtres vexés à revenir. "Si le mal et l'effroyable qui viennent de l'extérieur ont une telle signification pour la constitution des sphères humaines, c'est parce qu'elle rappelle [...] la constitution des capsules culturelles". II n'en reste pas moins que la libération de l'environnement nocif, ce repli dans la capsule de gâterie sonore qu'est l'île anthropogène, n'a jamais réussi à atteindre à l'immunité complète, et «l'enkystement culturel des sapiens" ne leur a jamais apporté "une liberté inconditionnelle à l'égard de la détresse, de la blessure". Le principe de distance a toujours été sapé par le principe d'invasion : invasion par les ancêtres et les revenants, dont on pouvait attendre l'apparition régulière dans le psychisme du groupe; invasion par catastrophes naturelles qui, sortant de l'environnement, faisaient et font encore irruption dans la physique du groupe; invasion par les nouvelles vérités qui ressortent des inventions et découvertes des innovateurs. (É 395-6) Invasion, invention et innovation : les trois fées qui ont présidé à la mise à mort des morts - c'est-à-dire au néant.

Car, dans la civilisation moderne, les méthodes d'acquisition de la connaissance se sont transformées suffisamment pour entraîner un changement fondamental dans le commerce avec les morts. Autrement dit, pour aboutir à une meilleure préservation du prin- 
cipe de distance, la civilisation moderne a trouvé une solution doublement efficace : inhumer ses morts autrement et se procurer ses vérités d'une autre manièré.

Si l'intérêt pour les choses de l'au-delà décline dans la modernité, c'est en premier lieu parce que l'on ne peut pratiquement plus avoir recours aux défunts pour obtenir des informations sur les choses à venir ; leur opinion ne serait pas d'un grand secours s'il s'agissait d'établir des règles techniques pour le futur management du monde. Le monde des vivants et le monde des morts sont devenus tellement dissemblables que les défunts n'ont plus de conseils à apporter aux vivants. À l'inverse, la capacité de poser aux morts des questions judicieuses s'est pratiquement éteinte parmi les contemporains. (É 405)

Le fait que, pour le travail scientifique, le détour par la transcendance est devenu superflu, a eu comme conséquence le congédiement des morts. Ils n'ont plus de tâche à accomplir pour leur descendance. Ils sont dépossédés du rôle thérapeutique qu'ils pouvaient jouer dans les coulisses du grand théâtre où évoluent les vivants. Ils ne sont plus les metteurs en scène qui permettaient aux vivants de dire : "Nous avons l'art afin de ne pas périr de la vérité." (PS 87)

On peut se demander : à quoi cela peut servir encore, la mémoire, et qu'est-ce que les vivants vont faire de leurs souvenirs, si les morts ne sont plus invités aux représentations théâtrales de la vie?

L'interruption du dialogue avec les morts a des effets qui vont bien au-delà des formules désincarnées affirmant la nature obsolète de la transcendance. Car le dialogue avec les morts fait partie d'un plus ample agencement, affectif et thérapeutique, renvoyant à la communication - dans l'espace et dans le temps - entre toutes les formes du vivant: "Selon la théorie de la philosophie de la nature formulée par Schelling, les organismes supérieurs, semblables à une mémoire vive somatique, conservent le souvenir intégral de leurs anciens modes d'être." Entre des êtres humains adultes, il semble pouvoir exister des relations qui ne sont compréhensibles que comme reproductions de relations "végétatives" de l'histoire antérieure. 
Non seulement tout organisme humain est un résultat et une mémoire stockée des processus de montée par paliers de I'histoire naturelle, depuis la pierre jusqu'à la créature vivante, sensitive et consciente d'elle-même; mais en chaque individu, on trouve aussi une mémoire qui conserve la propre histoire de son devenir depuis son époque utérine, et dans laquelle, dans des conditions exceptionnelles telle que celles induites par la cure magnétopathique, on peut aussi remonter de façon instructive. Cette possibilité de retour est la condition décisive du nouvel art de soigner. Les patients du magnétisme se "rappellent" pratiquement un état d'eux-mêmes dans lequel ils étaient animés et coordonnés depuis le centre de la mère, sous le mode d'un végétatisme extatique. (B269)

Si pour Schelling les êtres, les organismes supérieurs, sont euxmêmes semblables à une mémoire vive somatique, Benjamin montre que les souvenirs - et les souvenirs de l'enfance particulièrement ne sont que des invocations conjurant les morts à se manifester:

Berlin, prosaïque et bruyante, la ville du travail et la métropole de l'entreprise, possède, non pas moins mais davantage au contraire que beaucoup d'autres, de ces lieux et de ces instants où elle témoigne des morts, apparaît remplie par les morts, et cet obscur penchant pour ces instants, ces lieux, peut-être plus que tout le reste, donne aux souvenirs de l'enfance cet élément qui les rend à la fois si difficiles à saisir et aussi cruellement séduisants que les rêves à moitié oubliés. Car l'enfance, qui n'a pas d'idée préconçue, n'en a pas non plus à l'égard de la vie. L'enfant va à la rencontre du royaume des morts, là où celui-ci pointe dans le royaume des vivants, aussi précieusement lié à lui (et à vrai dire avec non moins de réserve) qu'à la vie même. (ÉA 281-2)

Heidegger n'est donc pas le seul, ni le dernier à croire que penser, donc, habiter le territoire du langage, équivaut à remercier les morts. Benjamin le soutient également, lorsque, dans sa Chronique berlinoise, il devient le médium des présences oubliées que son écriture ressuscite. ${ }^{15}$ Évoquer l'atmosphère de la ville qui était le territoire de l'enfance équivaut à accorder une existence "brève et imprécise comme une ombre" aux morts : «ils rasent ses murs comme des mendiants, ils surgissent à ses fenêtres comme des 
esprits pour disparaître aussitôt, ils prennent le vent sur les seuils comme un genius loci et même s'ils remplissent de leurs noms des quartiers entiers, c'est à la manière dont le nom d'un mort remplit la stèle sur sa tombe». (ÉA 281-2) ${ }^{16}$

Perdre leur importance est le destin commun des dieux et des morts, insiste Sloterdijk. "On se retourne vers eux comme vers des morts sans testament, vers des ancêtres dont on ne peut pas hériter grand-chose, dans le bien comme dans le mal - des batteries déchargées [...]". Les derniers morts vivants qui hantent leur descendance névrotique sont pris en charge par la psychanalyse, "qui a compris qu'elle est plus une entreprise de pompes funèbres interne pour les parents et les grands-parents qu'une forme médicale de la guérison». II reste la valeur d'usage des grands morts : en les faisant entrer comme des classiques dans la mémoire collective, on leur assigne le rôle d'assurer un passé commun à un groupe de civilisés. "Le passé sert désormais de camp de base depuis lequel la civilisation actualisée et futurisée se met en marche vers ses projets." (É 406) Malgré ces affirmations bien tranchées, dans Le penseur sur scène, Sloterdijk avait déjà invoqué la compagnie des morts d'une autre manière; une manière qui n'est pas sans rappeler la remémoration benjaminienne des espaces de l'enfance. Dans un premier temps, «[d]evant un objet classique, nous nous tenons, froids barbares, indifférents jusqu'à la moelle, et embarrassés, nous le retournons entre nos mains. Est-il encore bon à quelque chose?» Dans un premier temps, nous pouvons croire que la réponse est «non". Mais, quand nous pensons nous être débarrassés pour de bon de ces vieux objets, de ces vieux morts qui ne savent plus quoi répondre alors que nous leur posons des questions, «ils commencent lentement, mais irrésistiblement, à marcher derrière nous - non comme persécuteurs ni comme maîtres importuns mais comme ancêtres discrets et comme génies tutélaires, avec une générosité et une discrétion sur laquelle nous n'avions plus coutume de compter." (PS 12-13) Si, au moment du Penseur sur scène, la figure de Nietzsche avait permis à Sloterdijk de laisser (re)venir les génies tutélaires, dans Écumes c'est plutôt la proposition "sois toi-même un nouveau départ puisé dans tes propres forces!» qui le conduit. Avec des propositions de ce type, affirme-t-il, "on accomplit implicitement le passage de la théologie à l'immunologie - et eo ipso la libération conditionnée des égoïsmes finis». Car, au fond, c'était bien «le lieu empirique de l'égoïsme rénové et légitimé" qui constituait d'ordinaire le logement de Nietzsche, logement de celui «qui chercha vainement 
une place dans le monde qui lui soit supportable du point de vue atmosphérique". (É 477)

McLuhan a, lui aussi, donné un commentaire de l'«égoïsme rénové» dans son constat que «l'homme alphabétisé n'a plus besoin de considérer sa maison [...] comme une extension cultuelle de son corps". Son système immunitaire ne fait plus appel ni à l'univers, ni au fond divin, ni à des règles prétendument universelles. II n'est donc plus forcé d'établir une équivalence entre la maison et le cosmos. "L'habitant de la maison dans la modernité, soutenu par les médias, a remplacé les vagues systèmes de protection psychosémantiques de la métaphysique religieuse par ses cellules d'habitation spécialisées, à haut degré d'insulation juridique et climatique (et par les systèmes solidaires anonymes)". ( $E$ 477-8) Les medias ont, bien sûr, mis en place des lignes de communication - «solidaires" et "anonymes" destinées à remplacer le dialogue avec les morts. ${ }^{17}$

S'il est vrai que ce nouveau degré d'insulation, cette nouvelle immunité, permettent à l'homme moderne de se loger dans un espace où les invités indésirables n'ont jamais accès - mauvaises gens comme mauvaises nouvelles -, le mécanisme intégral de défense qu'est devenu le logement a pourtant ses points faibles, ses failles. II s'agit des situations où l'invasion intervient, justement, comme conséquence du haut degré d'étanchéité atteint par la machine immunitaire. Le symptôme de cette brèche porte le nom de dépression.

"Nous connaissons des situations dans lesquelles l'intellect parcourt une crise positiviste et perd momentanément la capacité de continuer à utiliser des informations. On constate tout d'un coup que tout n'est que ce que c'est, et rien d'autre. Dans cette position, le passage de l'être à la signification est bloqué - on n'a plus rien à dire sur quoi que ce soit.»18 Sloterdijk définit la dépression comme "une panne de la réceptivité - elle correspond à une perte de pression dans la chambre spatiale intérieure". (SM 301) Alors que "nous sommes faits pour produire constamment une folie de l'espace", dans le sens que Richard Wagner donnait au mot Wähnen, et qui veut dire à la fois délirer, supposer et rêver. Une "démence spatiale [...] intervient à chaque fois que l'âme s'installe dans une sphère suffisamment vaste pour elle, dans une dimension peuplée et qui respire. [...] [O]n ne voit à quel point cette expansion primitive est décisive qu'au moment où l'on est tombé dans la spatialité dépressive, ou plus précisément dans la privation d'espace». (SM 303) 
Dans le Livre des passages, ${ }^{19}$ Benjamin discute les caractéristiques de l'habitation ${ }^{20}$ au $\mathrm{XIX} \mathrm{X}^{\mathrm{e}}$ siècle, la manière dont cette forme originaire a fondamentalement changé au XXe, et quelles dépressions, quelle privation de l'espace ce changement a apporté avec lui. En premier lieu, dans l'habitation il faut voir «/'élément très ancien, éternel peut-être : le reflet du séjour de l'être humain dans le sein maternel». D'autre part, il faut considérer l'habitation "sous sa forme la plus extrême comme un mode d'existence du XIXe siècle". La vie non pas dans une maison, mais dans un boîtier qui porte l'empreinte de celui qui l'occupe, est la forme originaire de toute habitation. Le XIXe siècle aura considéré l'appartement comme "un étui pour l'homme; il a si profondément encastré celui-ci dans l'appartement, avec tous ses accessoires, que l'on croirait voir l'intérieur d'une boite à compas dans laquelle l'instrument est logé avec toutes ses pièces enfoncées dans de profondes cavités de velours le plus souvent violet. Est-il en effet un objet pour lequel le XIXe siècle n'ait pas inventé de boîtier et d'étui?» Par contre le XX siècle, "avec son goût pour la porosité, la transparence, la pleine lumière et l'air libre, a mis fin à la façon ancienne d'habiter. [...] Le modern style a ébranlé au plus profond d'elle-même la notion de boîtier. Aujourd'hui, elle a disparu et l'habitation a vu ses dimensions se réduire : pour les vivants, avec la chambre d'hôtel, et pour les morts, avec les crématoires". (LP 292-293)

Ce fragment nous ramène au journal par lequel a débuté notre commentaire, 21 à l'installation de fortune et au lien - inextricable pour Benjamin - entre la manière d'habiter et le rapport aux morts : les vivants réduits à rester à jamais des voyageurs sans correspondance, à se trouver un abri dans des chambres d'hôtel où aucune insulation, aucune immunité n'est plus possible; leurs morts, à finir d'une fin sans lendemain dans les crématoires, où tout espoir de devenir des génies tutélaires leur est enlevé.

C'est ainsi qu'on est arrivé à occuper cette position, à devenir ce personnage, ce voyageur sans correspondance. C'est ainsi que, malgré son rêve de nomade inoffensif, qui se voulait bon pâtre, le cosmopolite s'est retrouvé un jour impuissant - c'est-à-dire, incapable de rebrousser chemin - et assujetti irréversiblement aux exigences de la consommation. Sloterdijk parle des formes conduisant aux fonctions de gouverneur, de président, de chancelier, de cardinal, et oui, de professeur de philosophie, en termes d'ascension. II s'agit de fonctions ne pouvant être remplies que grâce 
à un "conditionnement mégalo-athlétique". Pour y arriver, il faut, en premier lieu, avoir coupé tout lien avec son enfance, "par un dressage et un entraînement de longue haleine arrachant le sujet à son domaine familier, l'aguerrissant, le fortifiant et l'élevant jusqu'à ce qu'il fonctionne au plus haut niveau". (MB 35) Ce n'est qu'ainsi qu'on devient un sujet véritablement souverain. Et pour qu'une telle souveraineté se maintienne, il faut sans hésitation «faire passer la rigueur». (MB 44)

Lorsque Benjamin dit que le XXe siècle, "avec son goût pour la porosité, la transparence, la pleine lumière et l'air libre, a mis fin à la façon ancienne d'habiter" (LP 292-293), nous y reconnaissons les conditions atmosphériques que le cosmopolite chérit au-dessus de tout : la pleine lumière, l'air libre. Effectivement, qui aujourd'hui voudrait encore vivre dans un boîtier? Qui voudrait se retrouver attaché aux habitudes qui nous tenaient enfermés dans une boîte à compas? Et surtout, qui voudrait encore penser à la tombe qui abrite cette boîte ultime, qui représente l'ultime domicile, la propriété immobilière sur laquelle on peut compter, qui sera la nôtre, où nous aurons notre éternité de quatre-vingt-dix-neuf ans?22 Autrement dit, les cosmopolites d'aujourd'hui ont appris comment ne pas retourner à l'enfance, ne pas faire de deuil. C'est bien ce que cela signifie, que de ne plus s'occuper des morts. La figure que Benjamin invoque, l'allégorie de la boîte à compas, renvoie directement à l'essence du processus de deuil, qui est de concevoir l'après-la-mort. Et de là, de penser la paix, l'éternité, le calme, la durée, telles que le promeneur solitaire les avait pensés dans son journal.

Le "surréalisme constitutif» au moyen duquel Sloterdijk désignait l'expansion de l'espace garantissait, justement, le bon éloignement nécessaire à tout individu (comme, par exemple, l'éloignement que permet une reproduction, comparé à la proximité imposée par un original). Pour le cosmopolite qui a compris que l'habitation «incarne l'unité de la géométrie et de la vie, l'utopie réalisée au niveau topique - comme projection intemporelle de l'intérieur", la maison devient complice des "besoins acosmiques. Elle forme une enclave de l'absence du monde dans le monde [...]. La maison qui est l'enveloppe d'un sommeil apporte la preuve la plus pure du lien entre l'immunité et le scellement de l'espace." (É 479) La question reste, toutefois, de savoir si cette immunité et ce scellement sauront le préserver, notre cosmopolite, des insomnies que peuvent lui causer la dépression, définie comme rétrécissement et privation de l'espace. 
Privation de l'espace, certainement, mais aussi rétrécissement des possibilités de communication avec ses proches, morts et vivants. Dans les mots de Benjamin : "Ainsi se perd le don de prêter oreille, et de ceux qui prêtent l'oreille la communauté disparaît." («Le conteur»125)

Sloterdijk offre une solution à la dépression du solitaire qui ne peut plus assurer la bonne distance entre lui-même et le monde environnant : cette thérapie s'appelle détente de l'espace et production d'horizon. II s'agit de ce qu'il reconnaît chez Nietzsche comme élément essentiel du bien-être physique, du rythme requis par une vie réussie : " la conviction de n'être qu'une incarnation, un porte-voix, le médium de forces supérieures" où aucun sens "supérieur» ne s'incarne. (PS 151) La philosophie romantique de l'organisme avait d'ailleurs déjà risqué cette "provocation" - qu'un objet de fascination, la sympathie, surgisse en même temps qu'un scandale, le sacrifice de l'autonomie - «au sens joyeux du terme, pour atteindre son objectif supérieur, la médiatisation de l'être humain sous forme de totalités globalisantes de la vie". (B 264-265) D'avantage encore, pour le Penseur sur scène, l'idée d'être médium et d'avoir la fonction d'un porte-voix n'était pas seulement une erreur superstitieuse. Dans «'irradiation civilisatrice du corps pour devenir parole», une influence, une contrainte et une séduction sont à l'œuvre qui ne proviennent pas du propre du locuteur et qui lui font dire des choses qu'il ne dit pas "de lui-même" au sens exact du terme. "Un "parler" essentiel est toujours en même temps une conformité avec un "entendre" - surtout avec un "avoir-entendu"» (PS 151-2) : "dire sa propre parole c'est, d'une façon heureuse, ne plus rien dire; remonter en arrière du logos; rejoindre la communicativité plus ancienne du vivant». (PS 154) Communicativité qui nous permettrait peut-être de reconstruire quelque chose comme une place dans le monde qui nous soit supportable du point de vue atmosphérique, et où le don de prêter oreille serait la première qualité des kosmopolites participants.

\author{
Angela Cozea \\ Études françaises, Université de Toronto
}




\section{Bibliographie des ouvrages cités}

Benjamin, Walter. Écrits autobiographiques. Trad. Christophe Jouanlanne et Jean-François Poirier. Paris : Christian Bourgois Editeur, 1994.

Gesammelte Schriften Band II,2 \& VI, Autobiographische Schriften, Francfort-sur-le-Main, Suhrkamp Verlag, 1985.

-. "Kitsch onirique», CEuvres II. Trad. Maurice de Gandillac, Rainer Rochlitz et Pierre Rusch. Paris : Gallimard, 2000.

-. "Le conteur. Réflexions sur l'œuvre de Nicolas Leskov», CEuvres III. Trad. Maurice de Gandillac, Rainer Rochlitz et Pierre Rusch. Paris : Gallimard, 2000.

. Paris Capitale du XIXe siècle. Le Livre des Passages. Trad. Jean Lacoste. Paris : Les Éditions du cerf, (1989) 1993.

Correspondance Adorno-Benjamin. Trad. Philippe Ivernel. Paris : La Fabrique éditions, 2002.

SloterdiJK, Peter. Bulles, Sphères, Microsphérologie, Tome I. Trad. Olivier Mannoni. Paris : Pauvert, 2002.

- Le penseur sur scène: Le Matérialisme de Nietzsche. Trad. Hans Hildenbrand. Paris : Christian Bourgois Editeur, 2000.

. Dans le même bateau Essai sur l'hyperpolitique. Trad. Pierre Deshusses. Paris : Éditions Payot \& Rivages poche, 2003.

-. Écumes Sphérologie plurielle. Trad. Olivier Mannoni. Paris : Maren Sell Éditeurs, «Hachette Littératures», 2005.

- La Domestication de l' tre. Pour un éclaircissement de la clairière. Trad. Olivier Mannoni. Paris : Éditions Mille et une nuits, 2000.

- Le penseur sur scène: Le Matérialisme de Nietzsche. Trad. Hans Hildenbrand. Paris : Christian Bourgois Editeur, 2000.

- Ni le soleil ni la mort. Jeu de piste sous forme de dialogue avec Hans-Jürgen Heinrichs. Trad. Olivier Mannoni. Paris : Fayard, 2003. 
1. Walter Benjamin, Écrits autobiographiques, traduction Christophe Jouanlanne et Jean-François Poirier, Paris, Christian Bourgois Editeur, 1994, p. 182 passim. Désormais, les références à cet ouvrage seront indiquées par le sigle $E A$, suivi du folio et placé entre parenthèses. Texte original : Gesammelte Schriften Band VI, Autobiographische Schriften, Francfort-sur-le-Main, Suhrkamp Verlag, 1985, p. 423. Désormais, les références à cet ouvrage seront indiquées par le sigle $A S$, suivi du folio et placé entre parenthèses.

2. Peter Sloterdijk, Dans le même bateau Essai sur l'hyperpolitique, traduction Pierre Deshusses, Paris, Éditions Payot \& Rivages poche, 2003, p. 53. Désormais, les références à cet ouvrage seront indiquées par le sigle $M B$, suivi du folio et placé entre parenthèses.

3. S'il fallait se tourner vers Sloterdijk pour apprendre ce que c'est que le journal intime, il nous dirait : «[L]'éclat individualiste [...] n'a pu devenir suggestif qu'au fil de l'évolution des médias dans les temps modernes. $Y$ ont surtout contribué les médias égotechniques qui, chez les individus, ont rôdé de nouvelles routines de retour à soi-même - en premier lieu les techniques d'écriture et de lecture à l'aide desquelles on a mis à l'épreuve des qualités historiquement nouvelles du dialogue intérieur, de l'examen de soi et de la documentation sur soi-même. Cela a eu pour conséquence le fait que l'Homo alphabeticus a non seulement développé des procédures singulières d'auto-objectivation, mais aussi des procédures de réunification avec soi-même par l'appropriation de l'objectivité. Le journal intime est une forme égotechnique de ce type, l'examen de conscience en est une autre." Peter Sloterdijk, Écumes Sphérologie plurielle, traduction Olivier Mannoni, Paris, Maren Sell Éditeurs, «Hachette Littératures», 2005, p. 517-518. Désormais, les références à cet ouvrage seront indiquées par le sigle $E$, suivi du folio et placé entre parenthèses.

4. Walter Benjamin, "Kitsch onirique», CEuvres II, traduction Maurice de Gandillac, Rainer Rochlitz et Pierre Rusch, Paris, Gallimard, 2000, p. 7-8.

5. Avec Peter Sloterdijk, je considère le rapport au paysage comme une des formes de réparation que l'être humain cherche, au sein des temps modernes, en d'autres mots, comme une figure de transfert. Car «nous ne transférons pas tant des affects incorrigibles sur des tierces personnes que des expériences précoces de l'espace sur de nouveaux lieux, et des mouvements primaires sur des théâtres lointains. Les frontières de ma capacité de transfert sont les frontières de mon univers." Cf. Bulles, Sphères, Microsphérologie, Tome I, traduction Olivier Mannoni, Paris, Pauvert, 2002, p. 15. Désormais, les références à cet ouvrage seront indiquées par le sigle $B$, suivi du folio et placé entre parenthèses.

6. Correspondance Adorno-Benjamin, traduite de l'allemand par Philippe Ivernel, Paris, La Fabrique éditions, 2002, p. 205-207. Dans sa présentation du volume, Enzo Traverso clarifie en quoi consistait, véritablement, la distance de onze ans qui séparait les deux hommes : "Les années vingt et trente avaient vu surgir l'oeuvre de Benjamin, de sa théorie de l'allégorie [...] aux aphorismes de Sens unique, de sa réflexion sur la perte de l'aura de l'œuvre d'art sous le capitalisme industriel à ses thèses sur la philosophie de l'histoire, de ses critiques littéraires à son projet inachevé sur Paris au XIXe siècle. L'œuvre d'Adorno commençait à peine à prendre forme à cette époque - elle n'éclora que dans l'après-guerre - et il ne faudra pas oublier, en lisant ces pages, qu'elles sont écrites d'un côté par l'auteur du Passagen-Werk et de l'autre, par le futur auteur de Minima moralia (1951) [...]. Au moment de la mort de Benjamin, en 1940, Adorno n'avait écrit qu'un livre sur Kierkegaard et quelques essais remarquables de critique musicale. Certains de ses 
travaux devaient beaucoup à la réflexion théorique de l'auteur de L'œuvre d'art à l'époque de sa reproductibilité technique, ce qu'Adorno reconnaissait d'ailleurs." ( $p$. 13)

7. Dans Le penseur sur scène: Le Matérialisme de Nietzsche, traduction Hans Hildenbrand, Paris, Christian Bourgois Editeur, 2000, p. 143-144 (désormais, les références à cet ouvrage seront indiquées par le sigle $P S$, suivi du folio et placé entre parenthèses), Sloterdijk décrit, sur un registre différent mais éclaircissant pour notre propos, «la vérité-coup de dents". II s'agit d' «une subversion dionysiaque de cet esprit de sérieux qui alourdit tant le monde moderne avec ses dominations d'idées théoriques et moralistes. Dans la corporéité langagière de Nietzsche, une nouvelle éthique - pouah! N'est-ce pas ainsi qu'on appelle cela aujourd'hui? de la pensée devait s'annoncer. La doctrine nietzschéenne du comportement intellectuel se recommande comme une hygiène ou comme une diététique - [...] comme une gymnastique mentale pour apprendre une éthique psychosomatique de l'intensité d'un genre nouveau. [...] [l]l sent qu'il n'y a rien de plus suspect que la peur de la vérité qui se fait passer pour de la conscience critique; et rien de plus faux que l'incapacité d'admettre qui se prend pour de la supériorité. Nietzsche développe surtout un sens hyperexcitable de l'obscénité de la prétendue communication entre sujets qui n'osent pas être présents dans leurs manifestations [...]. Le "démasquage" du christianisme comme mouvement du ressentiment et comme attentat historique contre la vie n'est probablement rien à côté de la révélation de la corporéité de la pensée." La "corporéité" est une caractéristique de la pensée de Benjamin en dépit de sa constitution humorale mélancolique. Sloterdijk y fait allusion implicitement en parlant du «moment de danger» où Benjamin reconnaissait tout moment historique essentiel : «Le danger étant autre chose qui médiatise toute subjectivité. Ainsi - à supposer qu'on ait quelque goût pour des formulations obscures - peut-on dire également : ce n'est pas le penseur qui agit et pense mais c'est le danger qui agit et pense en lui.» (48-49)

8. La traduction française, toujours vigilante et soucieuse de faire suffisamment abstrait, donne à l'expression der Bewegung selber ihren Stachel zu .nehmen le sens de "désamorcer le mouvement", alors qu'en allemand, à ce moment-ci, l'expression nous renvoie au moustique qui avait empêché le rêveur de s'abîmer dans le désir de l'éternité qui le hante chaque fois qu'il se trouve devant un paysage. J'aimerais par contre renvoyer ici au processus de conceptualisation qui aboutit au mot promptitude, à la «disposition (Bereitschaft) grandissante" de Benjamin'à s'enlever la vie - processus que je préfère désigner, avec lui, comme l'acte par lequel on réussit à «ôter l'aiguillon au mouvement».

9. Walter Benjamin, «Le conteur. Réflexions sur l'œuvre de Nicolas Leskov», CEuvres III, traduction Maurice de Gandillac, Rainer Rochlitz et Pierre Rusch, Paris, Gallimard, 2000, p. 139. Dans cette traduction remaniée, le titre «Le narrateur» a été remplacé par "Le conteur" pour éviter, j'assume, la méprise des ceux qui courent le danger - sans doute courant - de voir, dans l'art de raconter une histoire, une retombée empirique de la science appelée narratologie. Aussi, GS II, 2, p. 456.

10. Peter Sloterdijk, La Domestication de l' tre. Pour un éclaircissement de la clairière, traduction Olivier Mannoni, Paris, Éditions Mille et une nuits, 2000, p. 75. Désormais, les références à cet ouvrage seront indiquées par le sigle $D$, suivi du folio et placé entre parenthèses.

11. «La postmodernité est l'époque de l'“après-Dieu", celle qui fait suite aux empires classiques localement ouverts sur le monde.» (MB 56) 
12. Peter Sloterdijk, Ni le soleil ni la mort. Jeu de piste sous forme de dialogue avec Hans-Jürgen Heinrichs, traduction Olivier Mannoni, Paris, Fayard, 2003, p. 151. Désormais, les références à cet ouvrage seront indiquées par le sigle SM, suivi du folio et placé entre parenthèses.

13. "On doit cependant considérer comme une fonction vitale importante la capacité, pour un individu, de conserver une distance de sécurité à l'égard des personnes, des choses et des signes. Le principal type de production de distance consiste à faire quelque chose, intérieurement, de tout ce que l'on rencontre [...] à jouer ou à travailler avec - et à éviter par conséquent tout ce qu'on ne sait pas par quel bout prendre.» (SM 301)

14. Sauf si on vit et pense à une certaine distance du cosmopolitisme occidental : le superbe et atterrant film d'Aleksandr Sokurov, "Le deuxième cercle" (1990), qui porte sur une ancienne forme de prendre sur soi la peine et le devoir que nous cause le corps mort de notre proche, finit avec l'épitaphe : «Bienheureux nos plus proches et bien-aimés, pour être morts avant nous:» II est important de remarquer que le héros, dans ce film, est un jeune homme seul. La seule conversation qu'il a est avec l'agente des pompes funèbres. Et le lieu où sa solitude devient insupportabilité, où des hommes pareils à lui se transforment en ennemis qui cherchent à l'abattre, est un moyen de transports en commun.

15. Une des plus touchantes justifications de l'art nous est offerte par Sloterdijk dans le passage suivant : «ll y a donc quelque chose qui peut se voir, qui peut se montrer; ce quelque chose peut même - pour ainsi dire empiriquement en son propre nom - s'accepter et s'admettre. Le monde pourrait donc sombrer sans qu'un doute doive se lever sur ce que la simple perception de soi a dit à l'artiste au moment de son effort créateur : "c'était moi! Cela m'est arrivé! II fallait moi, comme médium, pour cet effet! Même si cela ne vaut pas grand-chose comme œuvre d'art - reste que ce qui parle en cette œuvre parlait à travers moi! Même si je suis éphémère reste que mon éphémérité disparaît dans ce qui, grâce à ma participation à un avènement de l'art, a réellement accédé à l'être"." (PS 95)

16. Pour une discussion plus détaillée de la manière dont moi-même je conçois le rapport entre l'écriture, la maison et le fait de remercier les morts, rapport que l'écrivaine Catherine Mavrikakis appelle la domestication des morts, je renvoie les lecteurs à l'entrevue «Rêvez pour moi» que nous avons réalisée ensemble sur le site de la revue Spirale http://www.spiralemagazine.com/radio spirale/03 reve.html

17. «... le postmoderne est un produit secondaire de la télécommande. Celle-ci constitue la technique clef pour le réglage de l'admission du son et de l'image, et eo ipso de la réalité dans l'égosphère. Si l'on considère qu'une créature du type Homo sapiens devient ce qu'elle écoute, le passage à l'auto-tuning optionnel des individus constitue une césure anthropologique : la contrainte extérieure et la contrainte intériorisée de l'écoute [...] se dissout dans la tendance à choisir soi-même son environnement auditif.» (É 525) Aussi : «Pour les médias audios, seul le téléphone [...] constitue l'un des moyens les plus efficaces pour assurer une liaison avec le monde depuis la réserve. [...] Compte tenu de cet effet d'immédiateté, il était légitime de décrire le téléphone comme un biophone - ce qui est moins qu'une vie ne peut pas appeler.» (É527)

18. Cette discussion de la dépression appartient à un dialogue fort instructif portant sur "ce qui se passe au juste lorsqu'un observateur, face à des reproductions, laisse aller son imagination et vit un processus créatif d'association d'idées, puis se tait devant les originaux." Plus particulièrement, il s'agit ici de la réaction de Michaux devant le Magritte original, l'homme et ses peintures. (SM 301 passim) 
19. Walter Benjamin, Paris Capitale du XIXe siècle. Le Livre des Passages, trad. Jean Lacoste, Paris, Les Éditions du cerf, (1989) 1993, p. 292-293. Désormais, les références à cet ouvrage seront indiquées par le sigle $L P$, suivi du folio et placé entre parenthèses.

20. «Le verbe wohnen (habiter) employé à la forme transitive - par exemple dans la notion de "gewohntes Leben" (la vie habituelle) - donne une idée de l'actualité frénétique qui se dissimule dans ce comportement. II consiste à donner forme pour nous à un boîtier.» (p. 293)

21. "21 juin : Au dernier jour de notre voyage de Marseille à Paris nous avons fait une halte en rase campagne. [...] [J]e me suis allongé sous un arbre. II y avait justement du vent; [...]. Tandis que je regardais le feuillage et suivais son mouvement, je me suis mis tout à coup à penser au grand nombre d'images, de métaphores de la langue qui nichent dans un seul et unique arbre. Ces branches, et avec elles la cime, se balancent hésitantes et plient en signe de refus, les branches, selon que le vent souffle, se montrent consentantes ou emportées, la masse des feuilles se cabre contre les insolences du vent, en frémit ou leur fait bon accueil, le tronc a une solide assise où il prend racine et une feuille porte ombrage à une autre. Complément à l'enquête de Brecht sur le mode d'habitation et les conceptions en général : habiter à l'hôtel. - La conception de la vie comme roman.» (ÉA 202-203)

22. La vogue extraordinaire que la crémation connaît dans la société «hautement civilisée" depuis quelques décennies s'auto-justifie au moyen d'un discours qui se montre soucieux de l'environnement d'un côté (il n'y a plus de place dans les cimetières pour tous les morts à venir, pour ne rien dire des problèmes d'hygiène que pose la décomposition des cadavres), critique des excès de l'industrie des pompes funèbres de l'autre. Dès que les parents sont morts, et pas un moment trop tôt, les cosmopolites les font incinérer et puis se mettent à débattre la question épineuse de quoi faire avec les cendres. Où les répandre de la manière la plus symbolique? Par quel moyen original, et en même temps économique, s'en débarrasser? Avec les cendres, toute la douleur de la perte est dissipée, et la célébration de la vie du défunt ou de la défunte peut commencer. On ne pense plus que les morts ont besoin de nos soins, non pas en tant que vivants dont on se rappelle; mais en tant que morts. II ne sera d'aucune manière question de les convoquer ou de chercher leur compagnie, pour ne rien dire de leur conseil. Ni de porter des fleurs, ni bleues ni rouges, sur leur tombe faite à présent de pleine lumière et d'air libre. Les vivants peuvent retourner à leur habitation provisoire, à la perfection des besoins acosmiques accomplis. 